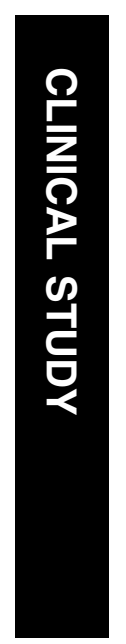

\title{
Proton beam therapy for iris melanomas
}

\begin{abstract}
Aims To describe the results in terms of local control, eye preservation and systemic evolution of iris melanomas treated by proton beam irradiation.
\end{abstract}

Methods Retrospective review of the charts of patients with iris melanoma treated by proton beam therapy between April 1998 and September 2002. Ciliary body melanomas with iris involvement or tumours with extrascleral invasion were excluded. Treatment consisted of $60 \mathrm{~Gy}$ of proton beam irradiation delivered in four fractions to the tumour volume.

Results A total of 21 patients were treated, median follow-up of 33 months (8-72 months). 15 patients presented a lesion with documented growth. The median clinical diameter was $5 \mathrm{~mm}(2-8 \mathrm{~mm})$, the median ultrasound diameter $4.8 \mathrm{~mm}(2-7.7 \mathrm{~mm})$ The patients were $6 \% \mathrm{~T} 1,57.1 \% \mathrm{~T} 2$, and $14.3 \% \mathrm{~T} 3$ all N0M0. The iridocorneal angle was invaded by the tumour in $\mathbf{7 1 . 4 \%}$ of patients. At the end of follow-up, all patients were alive with no proven metastatic disease except one patient with suspicious liver lesions. None of the patients showed tumour progression or ocular relapse. The tumour response at 2 years was a flat lesion for $6.3 \%$ of cases, partial regression in $\mathbf{7 5 \%}$ and stable in $\mathbf{1 8 . 8 \%}$. None of the patients required secondary enucleation. The main complication was cataract $(45 \%$ within 24 months of treatment). Raised intraocular pressure was observed in $15 \%$ of patients but no neovascular glaucoma.

Conclusions Proton beam therapy shows potential utility for selected cases of localised iris melanomas allowing excellent local tumour control and eye preservation. Further follow-up on larger series is needed to confirm these results.

Eye (2006) 20, 1300-1305. doi:10.1038/sj.eye.6702102; published online 18 Nobember 2005

Keywords: iris melanoma; proton beam therapy; radiation
L Lumbroso-Le Rouic ${ }^{1}$, S Delacroix ${ }^{2}$, R Dendale ${ }^{3}$, C Levy-Gabriel ${ }^{1}$, L Feuvret ${ }^{2}$, G Noel $^{2}$, C Plancher ${ }^{4}$, C Nauraye ${ }^{2}$, P Garcia², V Calugaru², B Asselain ${ }^{4}$ and L Desjardins ${ }^{1}$

\section{Introduction}

Malignant melanoma of the iris is a rare pigmented tumour with an estimated frequency of between 2 and $5 \%$ of all uveal tumours and represents the most frequent malignant tumour of the iris ${ }^{1,2}$ Most of the pigmented iris tumours have a benign course and may be observed, nevertheless several clinical features of the iris lesion (single nodular lesion, more than $3 \mathrm{~mm}$ in diameter, presence of intrinsic tumour vessels, associated with varying degrees of pigment seeding in the iridocorneal angle, presence of raised intraocular pressure, or episodes of hyphaema $)^{2-5}$ may help differentiate between a benign or a malignant lesion and should lead to treatment, in order to avoid the rare distant metastasis $(5 \%)$ described in this sub population of uveal melanomas. ${ }^{2}$ Treatment options depend on tumour size, location and the presence of any associated complications (pigment seeding in the iridocorneal angle away from the tumour, invasion of the iridocorneal angle). Enucleation is indicated for very extensive tumours, tumours with extrascleral extension and diffuse iris melanoma.

Conservative treatment for localised tumours can preserve a functional eye and may reduce the risk of metastasis. The various conservative treatment options include surgical resection, irradiation, or a combination of these two approaches. Cases of radioactive plaque irradiation, $^{6}$ and several cases of proton beam irradiation have been reported by various authors. ${ }^{4,7,8}$ However, limited data are available on proton beam therapy on this particular subset of uveal melanomas. We present the results of proton beam therapy for circumscribed iris melanoma with a median follow-up of 33 months.

\section{Materials and methods}

This is a single-centre retrospective study describing the treatment and outcome of patients with primary pigmented tumours of 
the iris treated by proton beam therapy. A computer search was conducted to identify the charts of patients corresponding to these criteria. Only patients treated for a primary iris lesion, excluding ciliary lesions with contiguous involvement of the iris, were included.

The diagnosis of iris melanoma was based on clinical and instrumental findings (conservative treatment without preliminary biopsy). The following patients were treated: patients with an old iris lesion that progressed during follow-up (increased volume or diameter) and a number of patients with a recent iris lesion whose clinical and complementary characteristics suggested a malignant tumour (single nodular lesion, more than $3 \mathrm{~mm}$ in diameter, presence of intrinsic tumour vessels, associated with varying degrees of pigment seeding in the iridocorneal angle, presence of raised intraocular pressure, or episodes of hyphaema). ${ }^{1,5}$

After a complete clinical ophthalmological examination including colour photography of the lesion by slit lamp, ultrasound examination (biometry, standard B mode ultrasound or UltraBioMicroscopy (UBM) when available in France at the time of diagnosis), and a general work-up looking for secondary lesions by abdominal ultrasound and chest X-ray, patients were referred to the proton therapy centre for irradiation. The presence of extrascleral extension and disseminated iris melanoma constituted a contraindication to this type of treatment.

In contrast with choroidal or ciliary lesions, clip placement was required in only one patient.

\section{Irradiation technique}

A three dimensional (3D) modelling of the tumour is based on the preliminary imaging examinations performed at the initial visit (photography, ultrasound). The dose distribution study is based on a 3D reconstruction of the eye and the main intraocular structures (optic disk, macula, lens, ciliary body, and cornea) using EYEPLAN software.

Reproducibility of treatment is ensured by a personalised contention system composed of a heatformed mask associated with a mouthpiece. This system provides a contention of the face of the patient in a sitting position.

The proton beam is fixed and horizontal. The patient fixes a luminous infrared diode with one eye. The position of gaze is determined in order to irradiate the tumour from its base and obtained by the dose distribution study. The perfect cooperation of the patient is needed to avoid the eye's movements. A $2.5-3 \mathrm{~mm}$ safety margin is included around the tumour volume (this tumour volume includes pigment seeding in the iridocorneal angle, when present at the time of diagnosis). The safety margin includes possible microscopic tumour extensions and the patient's possible eye movements. Irradiation is performed on a nondilated pupil, as for the pretreatment imaging. The eye position and possible movements during treatment are monitored during irradiation by an infra-red camera. The luminous diodes, creates corneal reflexes that can be monitored and projected on screens present inside and outside the treatment area. Once the eye placed, the iris, and the diode reflexions on the cornea are drawn on the screens. If the patient moves, the drawings do not correspond any more to the reflections and irradiation is stopped. The treatment is started again when the eye is in the right position. This is repeated at each treatment session. The technique allows detection of movements less than $1 \mathrm{~mm}$. The positioning of the eye and treatment are realised in a full-lit room and the diode light does not affect the iris contractions.

A personalised brass collimator is used to define the lateral limits of the irradiation dose to the target volume.

As eyelid irradiation can cause complications and ocular sequelae, the eyelids are excluded from the irradiation fields by means of retractors.

Irradiation is performed in the sitting position with topical anaesthesia (oxybuprocaine eye drops) allowing insertion of eyelid retractors.

The dose delivered is 60 Cobalt Gray Equivalent (CGE) in four fractions of $15 \mathrm{~Gy}$ over 4 days; the relative biological efficacy (RBE) for protons is equal to 1.1. Each fraction lasts between 45 and $70 \mathrm{~s}$. This dose is the one, which is currently used for the other uveal melanomas and has proved its efficacy.

A clinical follow-up is then ensured including clinical ophthalmological examination 1 month after irradiation, then every 3 months, alternatively by the ocular oncologist and the general ophthalmologist. Ocular ultrasound is repeated every six months for 2 years, then annually. A general follow-up for detection of metastases, based on 6-monthly hepatic ultrasound, is also performed.

The charts of these patients were reviewed retrospectively and the following baseline data were recorded: demographic (age, gender), ocular (visual acuity, eye colour, presence or absence of an old iris lesion, interval between onset of symptoms, and diagnosis), tumour (clinical diameter, presence or absence of an achromic lesion, site according to the central meridian of the lesion, presence of cataract, presence or absence of documented tumour growth, diameter and thickness on standard ocular ultrasound or $50 \mathrm{MHz}$ Ultra Biomicroscopy, presence of localised tumour invasion of the iridocorneal angle, presence of pigment seeding in the iridocorneal angle away from the tumour, presence of raised intraocular pressure, and 
response to treatment), irradiation parameters using the treatment plan established on the basis of the initial ocular and tumour data (including the dose received by the tumour, the maximum and minimum tumour diameters, thickness, tumour volume, distance of the tumour from the optic nerve and macula, percentage area or volume irradiated by 20,50 , or $90 \%$ of the dose delivered to the retina, internal surface of the eyeball, volume of the eyeball, lens, lens periphery, ciliary body, optic disk, and macula) and the local and general outcome. A complete functional assessment is performed annually with estimation of visual acuity, presence or absence of raised intraocular pressure and its response to treatment, systematic search for the following postradiation sequelae: palpebral sequelae, dry eye syndrome, keratitis, posterior synechiae, uveitis, iridial neovascularisation, hyphaema, cataract, and an 'other complication' item, which was described in detail. The clinical and instrumental tumour response and the new ultrasound tumour thickness were recorded. The tumour was classified as stable, presenting partial response (reduction of tumour thickness), complete response (flat lesion), or progression (increased tumour thickness or diameter)

\section{Statistical methods}

Statistical analysis was then performed on demographic data, tumour data, and follow-up data. A $\chi^{2}$-test was used to compare two percentages and a Student's $t$-test was used to compare two means. The limit of significance was $P<0.05$.

A methodology adapted to survival analysis was used to study mortality and local or systemic recurrence. Survival rates were calculated from the first day of treatment, corresponding to proton therapy to the date of the event considered (death, local relapse, metastases, and appearance of complications).

\section{Results}

From April 1998 to September 2002, 21 patients satisfying the selection criteria were treated.

The median follow-up was 33 months (range: 8-72 months). No patient was lost to follow-up.

\section{Baseline characteristics}

The mean age of the patients of this series was 51 years with a median age of 50 years (range: $17-82$ years). This series comprised of 12 female patients $(57.1 \%)$ and nine male patients $(42.9 \%)$. In all, $15(71.4 \%)$ of these patients presented an old iris lesion and six (28.6\%) presented a recent iris tumour.

The mean interval between onset of the first symptoms and the final diagnosis was 3.1 months (median: 2 months; range: 0-62 months), where 0 corresponded to asymptomatic patients $(42.9 \%)$.

A total of $71.4 \%$ of patients presented documented growth of an iris lesion. The tumour involved the left eye in $57.1 \%$ of cases and the right eye in $42.9 \%$ of cases. All lesions were unilateral. The eye colour was blue in $38.1 \%$ of patients, green in $28.6 \%$, brown in $23.8 \%$, and not specified in $9.5 \%$.

All lesions were pigmented, but contained an achromic component in seven patients (33.3\%) (Figure 1a). The tumour was essentially located in the lower quadrants, as only two patients presented a tumour in the upper quadrants. The tumour was in contact with the ciliary body in one patient. Tumour diameter and thickness are
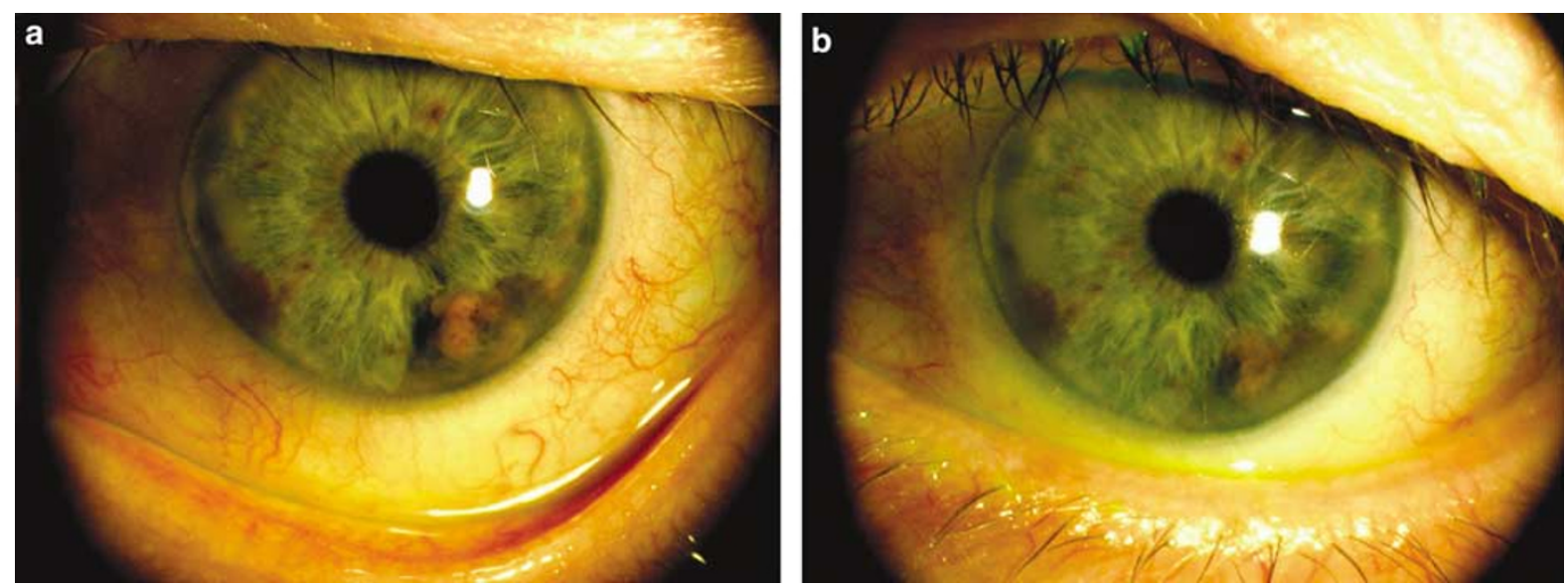

Figure 1 Iris melanoma with an achromic component before (a) and 2 years after proton beam therapy (b) showing regression of the lesion in thickness, particularly the achromic component. 
described in Table 1. (UICC criteria: $28.6 \% \mathrm{~T} 1,57.1 \% \mathrm{~T} 2$, and $14.3 \% \mathrm{~T} 3$, all patients were N0 M0 at diagnosis). Four patients present a localised cataract (19\%). The iridocorneal angle was touched by tumour in 15 cases (71.4\%). Ciliary body invasion was ruled out clinically and by ultrasound. Pigment seeding in the iridocorneal angle away from the tumour was observed in 10 patients $(47.6 \%)$. The seeding was estimated in degrees, ranging from 10 to $200^{\circ}$ with a mean of $74^{\circ}$ and a median of $30^{\circ}$. Raised intraocular pressure was observed in four patients, two of whom failed to respond to the medical treatment administered.

The mean baseline visual acuity was 20/30 (median: 20/25; range: 20/200-20/20).

\section{Clinical course}

All patients were alive at the end of follow-up. Only one patient presented multiple hepatic lesions suspected to be metastases, but not documented by liver biopsy (refused by the patient). The local tumour response was excellent for all patients, as no local recurrences have been observed to date. At 1 year after treatment, eight of the patients evaluated (40\%) have stable disease (lesion unchanged, no growth), and $12(60 \%)$ present partial regression (stable diameter but decrease of the thickness evaluated by UBM, especially for the achromic component, decrease of the intrinsic vessels when initially present). At 2 years, the lesion was flat in one

Table 1 Initial diameter and thickness of the tumour on clinical and ultrasound examination

\begin{tabular}{|c|c|c|c|c|}
\hline & $\begin{array}{c}\text { Number of } \\
\text { patients } \\
\text { evaluated }\end{array}$ & $\begin{array}{l}\text { Mean } \\
(\mathrm{mm})\end{array}$ & Median & Range \\
\hline Clinical diameter & 21 & 5.2 & 5 & $2-8 \mathrm{~mm}$ \\
\hline UBM ultrasound diameter & 7 & 5.18 & $4.8 \mathrm{~mm}$ & $2-7.7$ \\
\hline UBM ultrasound thickness & 7 & 2.87 & $2.3 \mathrm{~mm}$ & $1.10-6.70$ \\
\hline
\end{tabular}

Table 2 Ocular complications

\begin{tabular}{lccc}
\hline Complication & $\begin{array}{c}\text { Number of } \\
\text { patients } \\
\text { evaluated }^{\mathrm{a}}\end{array}$ & $\begin{array}{c}\text { Number of } \\
\text { patients } \\
\text { affected }\end{array}$ & $\begin{array}{c}\text { Percentage } \\
(\%)\end{array}$ \\
\hline $\begin{array}{l}\text { Cataract } \\
\text { Raised intraocular } \\
\text { pressure }\end{array}$ & 20 & 9 & 45 \\
$\begin{array}{l}\text { Anterior or posterior } \\
\text { synechiae }\end{array}$ & 20 & 3 & 15 \\
$\begin{array}{l}\text { Uveitis } \\
\text { Keratitis } \\
\text { Hyphaema }\end{array}$ & 20 & 1 & 10 \\
$\begin{array}{l}\text { anumber of patients evaluated: one patient does not have a follow-up of } \\
12 \text { months. }\end{array}$ & &
\end{tabular}

patient (6.3\%), showed partial regression for 12 patients (75\% of cases) (Figure 1a and b) and was stable for three (18.8\% of cases). For the six patients with a follow-up of at least 3 years, five have a partial response and one has a complete response (flat lesion). No patient has required secondary enucleation for ocular complications. The main postirradiation complications are described in Table 2.

No other complications were reported, especially no sicca syndrome, no photophobia, no iris neovascularisation, and no palpebral sequelae such as loss of eyelashes or depigmentation. The mean final visual acuity was 20/30 (median: 20/25; range: 20/200-20/20).

The main complication of treatment was cataract. Univariate analysis was performed to identify risk factors for the development of cataract. Male gender and the presence of an old iris lesion were found to be significant risk factors.

Irradiation parameters were then reviewed and lens opacities were observed even when the dose received by the lens was less than 12 Gy to less than $80 \%$ of the lens volume. Lens irradiation is difficult to avoid in view of the site of the tumour.

\section{Discussion}

Iris melanoma is a very rare tumour, as it represents only about $2 \%$ of all uveal tumours. ${ }^{1,2,5}$ The diagnosis of iris melanoma is highly probable in the presence of a combination of clinical characteristics suggesting a malignant tumour: single, unilateral lesion, measuring more than $3 \mathrm{~mm}$ in diameter, with abnormal intrinsic tumour vessels, pupillary deformity, cataract or pigment scatter, possibly associated with raised intraocular pressure. ${ }^{1,3,5}$ No biopsies confirming the malignant nature of the lesion were performed in this series of iris lesions, but the majority of patients nevertheless presented documented growth of a tumour arising on a pre-existing lesion, while all other patients presented a tumour whose initial clinical characteristics were highly suggestive of the diagnosis in agreement with the majority of authors, ${ }^{2,3,9}$ and according to the criteria defined above (essentially size). Conservative management by irradiation was therefore proposed on this body of evidence, by analogy with conservative radiotherapy ${ }^{10-17}$ performed for ciliary and choroidal melanomas, in order to avoid the metastasis and preserve a functional eye. Proton therapy is a modality of conformal radiotherapy, which uses a proton beam whose ballistic properties allow localised delivery of energy to the target volume. The depth of penetration of protons, directly related to their initial energy, can be very easily adjusted to the position of the tumour in the 
eye. A modulator system is used to deliver a homogeneous dose plateau over the thickness of the lesion. This homogeneous dose differs totally from the plaque therapy, which delivers a high dose gradient with a maximum dose at the surface of the plaque. In the iris tumours treatments the highest dose is therefore, received by the cornea when using a plaque.

Proton beam irradiation has been rarely reported for iris lesions ${ }^{4,18}$ and, to our knowledge, has not been the subject of specific publications. Several cases of iris lesions treated by proton beam therapy have been mentioned in series presenting the results of treatment for uveal melanoma, but these studies do not detail the local outcome of this subset of melanomas and their course after treatment is therefore not analysable. ${ }^{8,19,20}$

The results of this short series of patients are very encouraging, as patient survival was excellent with only one patient with suspected metastatic melanoma. Iris melanomas are known to have a slow course with a low-

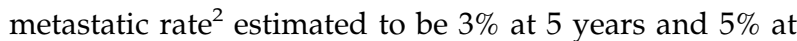
10 years but this series presents relatively bigger sized lesions (median diameter and thickness comparable to our series but the range is wider and they include patients with extrascelaral invasion) than the ones we describe, all patients treated in our centre presented small, circumscribed lesions with no extrascleral extension. The results in terms of local control are also very favourable, as no cases of progression or local recurrence have been observed with a follow-up of 33 months. Conway et $a l^{9}$ reported a recurrence rate after surgical resection of about $4 / 44$ patients, occurring an average of 3 years after surgery. Another study reported no relapse after a follow-up of 31 months for 11 resections. ${ }^{21}$ In a series of 28 cases treated by iridectomy, Jensen $^{22}$ reported three recurrences 5-7 years after surgery.

As all lesions treated in our series were localised and relatively small, conservative surgical management would have constituted a suitable alternative treatment. However, irradiation was proposed in order to minimise postoperative complications, as the iridocorneal angle was invaded in the majority of cases and wide resection with cyclectomy would have been necessary with a risk of more severe immediate or long-term haemorrhagic, infectious and functional complications than those related to circumscribed irradiation. Surgical resection is associated with a risk of immediate haemorrhagic or infectious complications, or, in the longer term, may cause raised intraocular pressure and bothersome photophobia in the absence of pupillary reconstruction. ${ }^{9}$ Furthermore, the initial management of the tumour did not appear to be a risk factor for the development of metastases in the study by Shields et $a l^{2}{ }^{2}$ who propose surgical resection in $60 \%$ of their patients with iris melanoma.

${ }^{125}$ I radioactive plaque irradiation was proposed by Shields et $a l^{6}$ for particular cases of iris tumours, for which surgery was not indicated or after a first recurrence. They reported a local recurrence rate of $15 \%$ at 10 years and $8 \%$ at 5 years, but also a considerable complication rate, comprising keratitis, neovascular glaucoma, and a secondary enucleation rate of up to $13 \%$ at 5 years and $21 \%$ at 10 years, although the authors do not directly attribute these enucleations to irradiation. However, the tumours treated by this modality were more extensive than the lesions of our series, with a median diameter of $7 \mathrm{~mm}$.

The main complication of proton beam irradiation is the relatively rapid appearance of cataract (45\% with a median follow-up of 33 months). All patients in whom cataract induced a loss of visual acuity were able to be operated with good functional recovery. Cataract is also the most frequent complication in the series published by Shields et $a l^{6}{ }^{6}$ as they report a 5-year cataract rate of up to $70 \%$. The minimum dose at which cataract occurred could not be defined and it appears difficult to irradiate all of the tumour volume without also irradiating the lens, in view of the position of this type of tumour.

The second most frequent complication, which can threaten the patient's visual prognosis is raised intraocular pressure. However, it occurs less frequently, in $15 \%$ of treated patients, not all corresponding to those patients with preoperative raised intraocular pressure. The exact pathophysiology of raised intraocular pressure remains unclear, but may involve invasion of the iridocorneal angle by tumour cells and by the tumour as well as postirradiation phenomena. Only one of the three patients with raised intraocular pressure in our series only partially responded to medical treatment.

The cost of proton therapy (which used to be very expensive) is not anymore a major problem, currently in France the price of the whole proton treatment is 1968 euros, plaque therapy and surgery prices depending on the hospital stay of the patient: the cost is 1243 euros per day, which makes these last two treatments more expensive as they often need at least two or three days of hospital stay.

Proton beam therapy therefore appears to be a valuable alternative treatment to wide surgical resection for relatively small iris melanomas. The tumour response is satisfactory with an acceptable complication rate and treatment does not induce any visual deterioration. A longer follow-up is required to evaluate the long-term efficacy of this treatment modality in view of the long natural course of this type of tumour. 


\section{References}

1 Shields J, Shields C. Melanocytic tumors of the iris stroma In: Shields J, Shields C (eds.). Intraocular Tumors A Text and Atlas. Saunders, 1992, pp. 69-77.

2 Shields CL, Shields JA, Materin M, Gershenbaum E, Singh $\mathrm{AD}$, Smith A. Iris melanoma: risk factors for metastasis in 169 consecutive patients. Ophthalmology 2001; 108(1): 172178. Order.

3 Harbour JW, Augsburger JJ, Eagle Jr RC. Initial management and follow-up of melanocytic iris tumors. Ophthalmology 1995; 102(12): 1987-1993.

4 Damato B. Uveal melanoma. In: Damato B (ed). Ocular Tumours. Oxford: Butterworth-Heinemann, 2000, pp. 57-93.

5 Marigo FA, Finger PT. Anterior segment tumors: current concepts and innovations. Surv Ophthalmol 2003; 48(6): 569-593. Order.

6 Shields CL, Naseripour M, Shields JA, Freire J, Cater J. Custom-designed plaque radiotherapy for nonresectable iris melanoma in 38 patients: tumor control and ocular complications. Am J Ophthalmol 2003; 135(5): 648-656.

7 Gupta M, Puri P, Rennie IG. Iris seeding following iridocyclectomy for localised iris melanoma. Eye 2001; 15(Part 6): 808-809.

8 Spatola C, Privitera G, Raffaele L, Salamone V, Cuttone G, Cirrone $\mathrm{P}$ et al. Clinical application of proton beams in the treatment of uveal melanoma: the first therapies carried out in Italy and preliminary results (CATANA Project). Tumori 2003; 89(5): 502-509.

9 Conway RM, Chua WC, Qureshi C, Billson FA, Harbour JW, Augsburger JJ et al. Primary iris melanoma: diagnostic features and outcome of conservative surgical treatment. $\mathrm{Br}$ J Ophthalmol 2001; 85(7): 848-854.

10 Char DH, Kroll SM, Castro J. Ten-year follow-up of helium ion therapy for uveal melanoma. Am J Ophthalmol 1998; 125(1): 81-89.

11 Diener-West M, Earle JD, Fine SL, Hawkins BS, Moy CS, Reynolds SM et al. The COMS randomized trial of iodine 125 brachytherapy for choroidal melanoma, III: initial mortality findings. COMS Report No. 18. Arch Ophthalmol 2001; 119(7): 969-982.
12 Egger E, Zografos L, Munkel G, Bohringer T, Bercher L, Chamot L. Results of proton radiotherapy for uveal melanomas. Front Radiat Ther Oncol 1997; 30: 111-122.

13 Fuss M, Loredo LN, Blacharski PA, Grove RI, Slater JD. Proton radiation therapy for medium and large choroidal melanoma: preservation of the eye and its functionality. Int $J$ Radiat Oncol Biol Phys 2001; 49(4): 1053-1059.

14 Gragoudas ES, Seddon JM, Egan K, Glynn R, Munzenrider $\mathrm{J}$, Austin-Seymour $\mathrm{M}$ et al. Long-term results of proton beam irradiated uveal melanomas. Ophthalmology 1987; 94(4): 349-353.

15 Gunduz K, Shields CL, Shields JA, Cater J, Freire JE, Brady LW. Plaque radiotherapy of uveal melanoma with predominant ciliary body involvement. Arch Ophthalmol 1999; 117(2): 170-177.

16 Zografos L, Perret C, Gailloud C. Conservative treatment of uveal melanoma by accelerated proton beam. In: Bornfeld N, Gragoudas E, Höpping W, Lommatzsch P, Wessing A, Zografos L (eds). Tumors of the eye. Proceedings of the second international symposiumon diagnosis and treatment of intraocular tumors, Geneva, Switzerland, November 23-23 1987. Amsterdam/New York: Kugler publications, 1991, pp. 497-506.

17 Wilson MW, Hungerford JL. Comparison of episcleral plaque and proton beam radiation therapy for the treatment of choroidal melanoma. Ophthalmology 1999; 106(8): 1579-1587.

18 Zografos L. Mélanomes iriens. In: Zografos L (ed). Tumeurs Intraoculaires. Paris: Masson, 2002, pp. 291-302.

19 Gragoudas ES, Egan KM, Seddon JM, Walsh SM, Munzenrider JE. Intraocular recurrence of uveal melanoma after proton beam irradiation. Ophthalmology 1992; 99(5): 760-766.

20 Gragoudas ES, Seddon J, Goitein M, Verhey L, Munzenrider $\mathrm{J}$, Urie $\mathrm{M}$ et al. Current results of proton beam irradiation of uveal melanomas. Ophthalmology 1985; 92(2): 284-291.

21 Cardine S, Labetoulle M, Kirsch O, Di Nolfo M, Offret H, Frau E. Iris melanomas. A retrospective study of 11 patients treated by surgical excision. J Fr Ophtalmol 2003; 26(1): 31-37.

22 Jensen OA. Malignant melanoma of the iris. A 25-year analysis of Danish cases. Eur J Ophthalmol 1993; 3(4): 181-188. 